\title{
Investimento Estrangeiro Direto e o Setor de Serviços de Telecomunicações Brasileiro
}

\author{
Foreigner Direct Investment and \\ Brazil's Telecommunications Sector
}

\author{
Jefferson Nery do Prado* \\ Elaine Aparecida Fernandes** \\ Silvia Harumi Toyoshima** * \\ Evaldo Henrique da Silva****
}

\begin{abstract}
Resumo: O objetivo do presente estudo é analisar a capacidade de inovação tecnológica das empresas nacionais e estrangeiras no setor de serviços de telecomunicações. Para a apuração dessa capacidade, esta pesquisa elabora o índice de capacidade de inovação por meio dos microdados da Pesquisa de Inovação Tecnológica. Constata-se que não há distinção entre intensidade de inovação de empresas nacionais e estrangeiras, que apresentam índices médios de 0,4265 e 0,4196, respectivamente. Desse modo, conclui-se que a atividade de geração de inovação no Brasil (considerando empresas nacionais e estrangeiras), medida pelas atividades de pesquisa e desenvolvimento (PED) desenvolvidas internamente na empresa, não ocorreu nem para adaptação de inovações geradas externamente, o que é preocupante, pois atualmente são necessários gastos em PEBD significativos para apenas imitar.
\end{abstract}

Palavras-chave: Inovação. Serviços de telecomunicações. Investimento direto.

Abstract: The purpose of the present study was to analyze the capacity of technological innovation from domestic and foreign firms in the telecommunications sector. To estimate the capacity for technological innovation, the index of capacity for innovation was created, based on micro data of "Pesquisa de Inovação Tecnológica". The data showed no distinction between innovation intensity in either domestic or foreign firms, which presented average indexes of 0.4265 and 0.4196 , respectively. In this context, it is concluded that the activity of innovation generation in Brazil (considering domestic and foreign companies), as measured by $\mathrm{R} \mathcal{E} \mathrm{D}$ activities internally developed in the company, there was not for adaptation of externally generated innovations, which is worrying, because currently are necessary expenses in significant $\mathrm{R} \mathcal{E} \mathrm{D}$ only to imitate.

Keywords: Innovation. Telecommunications services. Direct investment.

* $\quad$ Doutor em Economia Aplicada pela Universidade Federal de Juiz de Fora (UFJF). Professor da Universidade Anhembi Morumbi. E-mail: jnprado@yahoo.com.br

* $\quad$ Doutora Economia Aplicada pela Universidade Federal de Viçosa (UFV). Professora da Universidade Federal de Viçosa (UFV). E-mail: eafernandes@ufv.br

*** Doutora em Ciência Econômica pela Universidade Estadual de Campinas (Unicamp). Professora aposentada da Universidade Federal de Viçosa (UFV). E-mail: htsilvia@ufv.br

**** Doutor em Economia da Indústria e da Tecnologia pela Universidade Federal do Rio de Janeiro (UFRJ). Professor na Universidade Federal de Viçosa (UFV). E-mail: ehsilva@ufv.br 
JEL Classification: F23; O33.

\section{Introdução}

Dada a importância dos serviços de telecomunicação para o desenvolvimento nacional e a grande participação do capital estrangeiro no setor, a questão que se coloca neste presente trabalho é: empresas nacionais e estrangeiras diferem em termos de comportamento inovador? Nesse sentido, é importante ressaltar que as empresas estrangeiras são grandes difusoras de processos inovativos de suas matrizes e podem não gerar diretamente inovação na economia receptora se não tiverem adequada capacidade de absorção (NARULA; ZANFEI, 2004). Essa realidade é particularmente verdadeira para o setor de serviços de telecomunicação brasileiro. Nesse setor, segundo dados da Pesquisa de Inovação Tecnológica (Pintec) (IBGE, 2005), o gasto em máquinas e equipamentos, por exemplo, foi maior que o gasto em Pesquisa e desenvolvimento (PED), tanto para as empresas nacionais, quanto para as estrangeiras. No entanto, dadas as condições inadequadas da pesquisa científica no Brasil, investir em PED pode ser um processo dispendioso e arriscado, fazendo com que o investimento não resulte em retorno esperado para as empresas, sejam nacionais ou estrangeiras. Seria esperado que a entrada de empresas de fora do país alterasse o comportamento tanto dos implementadores de políticas públicas quanto das empresas nacionais, de forma a absorver, ao máximo, tecnologias de fronteira desenvolvidas externamente.

Diante desse contexto, procurou-se determinar se esse capital externo, que migrou para o setor de telecomunicações brasileiro, teve como objetivo apenas explorar um mercado ainda incipiente ou, de fato, investir em empresas de telecomunicações de modo a intensificar e disseminar inovações no setor. O presente estudo busca analisar o efeito da entrada de investimento estrangeiro direto (IED), em termos de inovação tecnológica, no setor de serviços de telecomunicação brasileiro. Especificamente, pretende-se: a) construir um índice de capacidade de inovação para as empresas nacionais e estrangeiras do setor de serviços de telecomunicação; b) comparar empresas nacionais e estrangeiras em termos de comportamento inovador; e c) verificar se o capital migrou para apenas explorar mercado interno ou foi agente ativo no investimento em inovação tecnológica no setor.

A abordagem teórica que subsidia a presente análise está fundamentada em Hymer (1976) e sistematizada em Dunning (1981, 1988, 1999). Esses autores procuram identificar as principais razões para a existência do IED. Dentre os prováveis motivos, aproveitar os recursos naturais, humanos, tecnológicos, entre outros, e/ou aproveitar o mercado interno podem ser citados como exemplos. Cantwell (1989) e Narula e Zanfei (2004) também são utilizados como fontes teóricas importantes, pois fazem a ligação entre a existência de empresas estrangeiras e o seu desempenho tecnológico. 
Alguns trabalhos abordam temas semelhantes. Li e Xu (2004) analisam o impacto da privatização e da competitividade das empresas no setor de telecomunicações em alguns países do mundo, concluindo que houve melhor alocação de trabalho e capital, expansão dos serviços prestados e aumento na produtividade total dos fatores. Galina e Plonski (2005) verificam se as subsidiárias brasileiras, de empresas multinacionais, estavam de fato contribuindo para o desenvolvimento de tecnologia para o setor de telecomunicações em âmbito internacional, por meio de patentes e dados bibliométricos. Sua principal conclusão é que o Brasil está muito abaixo nesse item se comparado a outros países como Índia, Israel e China. Cuilenburg e Slaa (1995) pesquisam em que medida há competição entre as empresas de serviços de telecomunicações e se a inovação tecnológica é desenvolvida na direção do interesse público. Esses autores concluem que existe uma correlação positiva entre competição e inovação nas telecomunicações quando se trata de economias desenvolvidas, mas o mesmo não ocorre em economias em desenvolvimento. Nestas, o governo precisa intervir para corrigir as falhas de mercado. Islam, Fiebig e Meade (2002) analisam a inovação tecnológica no setor em diversos países do mundo, utilizando os dados de telefonia digital, concluindo que o modelo pooling, combinado com o modelo de difusão de Gompertz, ajusta-se melhor para analisar a inovação do setor de telecomunicações. Madden e Savage (1999) mensuram o ganho na produtividade total dos fatores e estimam a inovação tecnológica por meio da concentração de empresas no setor, da privatização e do tamanho do mercado.

Existe vasta literatura que trata do tema, mas ainda não foi elaborado um índice de inovação que utilize os microdados da Pintec como ferramenta de análise. Além disso, dada a dificuldade em se trabalhar com esse tipo de dado, não há um grande número de trabalhos que tenha se utilizado de informações em nível de empresa no Brasil. O presente estudo compara e analisa os indicadores de cada empresa do setor de serviços de telecomunicações, o que confere maior precisão na análise sobre o tema abordado.

Este artigo, além desta introdução, está organizado em mais quatro seções: a segunda resume as teorias que fornecem o suporte teórico do estudo; a terceira expõe o método de análise; a quarta ilustra os resultados; e, por fim, a quinta traça as conclusões.

\section{Investimento Direto Estrangeiro e Inovação}

Esta seção resume as principais teorias que dizem respeito ao IDE e à inovação. Autores como Dunning, Hymer, Schumpeter, Nelson e Winter, Pavitt, Dosi, entre outros, são discutidos a seguir. 


\subsection{Investimento Estrangeiro Direto}

No início da década de 1960, havia o predomínio da teoria financeira neoclássica de fluxo de portfólio para explicar os movimentos de capitais internacionais. Seus trabalhos tinham por axioma que os fluxos de investimento estrangeiro ocorriam sob a condição de um ambiente em concorrência perfeita, sem custos de transação, tendo como base apenas a remuneração do capital que seria empregado (DUNNING; RUGMAN, 1985).

Em 1960, a tese de doutorado ${ }^{1}$ de Stephen H. Hymer, apresentada no Massachusetts Institute of Technology (MIT), quebrou o paradigma sustentado pela teoria neoclássica, enfocando os movimentos de capital estrangeiro sob a ótica das empresas multinacionais (EMNs). Tal enfoque subdividiu os estudos sobre o tema, e os trabalhos sobre essa nova subdivisão seguem o que a literatura denomina de internalização da produção, fornecendo um respaldo microeconômico a um tema abordado, até então, sob o ponto de vista macroeconômico.

Segundo Hymer (1960), as empresas multinacionais são uma criação das imperfeições de mercado, pois têm a habilidade de usar suas operações internacionais para separar mercados e remover competidores ou, ainda, explorar uma vantagem específica.

Toda a análise de Hymer (1960) é baseada em imperfeições estruturais em que uma EMN possa melhorar um ativo para a própria empresa. Isso inclui economias de escala, vantagens de conhecimento, distribuição de contratos de trabalho, diversificação na produção e vantagens de crédito. Todos esses aspectos ${ }^{2}$ ajudam as EMNs a aumentarem sua participação ou, muitas vezes, conquistarem posição de liderança nos mercados em que atuam.

Uma das grandes vantagens de uma EMN está na habilidade de usar mercados internos em todo o mundo. Tais empresas podem usar preços de transferência, manobrar ativos, mover instalações produtivas e realizar demais práticas que irão prover um maior grau de liberdade em comparação a uma firma nacional confinada em um único país. Embora, como contraponto, as EMNs se deparem com ambientes de incerteza, causados por governos estrangeiros que podem mudar subitamente sua política econômica ou até mesmo sofrer influência de aspectos culturais e sociais particulares a cada país, tais aspectos podem determinar a eficiência econômica e sua aceitação em outros países.

Segundo Hymer (1960), as EMNs dispõem de vantagens de natureza - principalmente tecnológica - que lhes permitem atuar nos mercados externos caracterizados pela concorrência imperfeita. As imperfeições mencionadas pelo autor

$1 \quad$ Foi publicada apenas em 1976.

2 Os aspectos citados no texto e a maneira como podem ser combinado de modo a fechar mercados estão descritos em detalhes na obra de Bain (1956). 
correspondem fundamentalmente às que foram analisadas por Bain (1956): incertezas nos preços das matérias-primas ou componentes; longa duração do processo de fabricação; e ser comprador único. Quando investem em filiais de produção no exterior, as empresas oligopolistas utilizam vantagens para reduzir as incertezas do mercado. A manutenção do controle sobre filiais externas não passa necessariamente pela propriedade majoritária do capital, mas é um fator fundamental - que diferencia os investimentos diretos das outras operações internacionais - para a preservação das vantagens e eliminação dos potenciais concorrentes (DUNNING, 2010).

A teoria eclética, cujo principal representante é Dunning (1981, 1995, 1999, 2000, 2001, 2002, 2006, 2008), se baseia nas teorias da organização industrial e da locacional para explicar a existência de IDE. A abordagem de Dunning entende que determinadas falhas de mercado, como custos de informação e transação, induziriam uma empresa a optar pelo investimento direto em vez de exportação e licenciamentos a outras empresas.

Nesse sentido, uma empresa decide pela produção internacional se tiver vantagens diferenciais (paradigma OLI). A vantagem de propriedade é um exemplo importante e está relacionada à existência de ativos específicos. Dotações nacionais de fatores passíveis de serem internalizados por empresas e tecnologias produtivas são alguns desses ativos considerados primordiais. Outro exemplo são as vantagens de localização como recursos humanos e naturais abundantes, know how tecnológico, carga tributária, infraestrutura, entre outros. Por último, há as vantagens de internalização que resultam de imperfeições de mercado. Dunning considera esse tipo de vantagem como o principal fator a estimular, em nível internacional, as integrações vertical e horizontal das firmas.

Após a decisão de produção internacional, Dunning sintetiza algumas classificações que podem surgir das diferentes combinações das três vantagens supracitadas. Assim, o investimento direto pode ser do tipo resource seeking, market seeking, asset seeking e efficienty seeking. O primeiro tipo é aquele investimento que tem por objetivo produzir bens para a exportação com a aquisição de recursos específicos a baixos custos (tem pouco efeito sobre a economia receptora). No segundo tipo, as empresas estão dispostas a ofertar bens e serviços também para o mercado interno do país receptor, assim como para países vizinhos. Esse tipo implica em maior aprendizagem, pois a tecnologia recebida da matriz precisa ser adequada às peculiaridades do mercado em que se encontra a filial. Em adição, tem-se o terceiro tipo, asset seeking. Esse tipo de investimento tem como foco principal a compra de empresas existentes. Fusões, aquisições e joint-ventures podem ser citadas como exemplos. A quarta e última estratégia, efficiency seeking, tem como objetivo principal o aproveitamento do mercado doméstico, entretanto o foco é a produção e exportação a outras filiais da corporação. 
Portanto, o capital estrangeiro não leva em conta apenas a remuneração que irá obter, mas também como as empresas receptoras desse capital se organizam, planejam, financiam, produzem sistemática e permanentemente conhecimentos técnicos e desenvolvem expertise que lhes darão vantagens competitivas. Observa-se que o investimento em PEBD tem papel fundamental em orientar os fluxos de capital externo em qualquer que seja o país ou mercado de interesse.

\subsection{Inovação Tecnológica}

O conceito de inovação tecnológica utilizado na presente pesquisa parte das definições schumpeterianas e neoschumpeterianas para o que é chamado de inovação em termos de produto e de processo. Especificamente, tais definições estão presentes no Manual de Oslo (1997), que serviu como base para a elaboração da fonte de dados, a Pintec 2005 (IBGE, 2005) utilizada neste estudo.

Inovação tecnológica passou a figurar como um importante fator no estudo do desenvolvimento econômico a partir de Schumpeter (1982). ${ }^{3}$ Para o autor, as inovações de modo geral, incluindo a tecnológica, podem ser conceituadas como a implementação de algo novo, inexistente no setor produtivo, e que resulta da combinação dos diversos insumos de maneira diferente. Tais inovações provocam mudanças espontâneas e descontínuas nos fluxos de renda, associadas às alterações permanentes no ponto de equilíbrio, no qual a economia se encontra, desempenhando o papel de motor do desenvolvimento econômico.

A partir desse raciocínio, pode-se elencar os tipos de inovação identificados por Schumpeter (1982): a) introdução de um novo bem que os consumidores ainda não estiverem familiarizados ou de uma qualidade diferente; b) introdução de um novo método de produção que ainda não tenha sido introduzido na esfera produtiva, podendo consistir também em uma nova maneira de manejar comercialmente uma mercadoria; c) abertura de um novo mercado em que o ramo particular da indústria de transformação do país em questão não tenha ainda entrado, quer esse mercado tenha existido antes ou não; d) conquista de uma nova fonte de oferta de matérias-primas ou de bens semimanufaturados, independentemente do fato de que essa fonte já exista ou deva ser criada; e e) estabelecimento de uma nova organização de qualquer indústria, como a criação de uma posição de monopólio ou a fragmentação de uma posição de monopólio.

Segundo o autor, o processo inovativo gera um efeito em que os elementos novos passam a substituir os antigos, num processo endógeno, e tal substituição é determinante para se entender o capitalismo e como as empresas devem se adaptar a essa mudança para que possam sobreviver em seu mercado de atuação. Tal efeito, Schumpeter (1961) denomina de destruição criativa.

3 A primeira edição data de 1911. 
Dentre os seguidores de Schumpeter, encontra-se a escola denominada evolucionária, ou neoschumpeteriana, que incorporou elementos da teoria evolucionária da biologia a seus pressupostos teóricos. Segundo Nelson e Winter (1982), a teoria evolucionária faz uma analogia da competição entre empresas com a competição entre espécies, de modo que as mais aptas sobrevivem no mercado. Os conhecimentos tácitos de uma empresa corresponderiam a seus genes, a partir dos quais ela tenta sobreviver no mercado por meio de um processo contínuo de inovações.

Uma das premissas fundamentais dessa visão é que os agentes são dotados de racionalidade limitada e, portanto, a economia não caminha naturalmente para o equilíbrio, mas vive em um ambiente de incerteza. Nesse ambiente, a principal tarefa das empresas não consiste na maximização de lucros, mas em elaborar um conjunto de rotinas para que possam tomar a melhor decisão possível diante das várias possibilidades de escolha, mas cujos resultados são desconhecidos $a$ priori. Com base nos dados que possuem, procuram traçar uma estratégia de ação, visando atingir determinado objetivo, que pode ser de manutenção de liderança no mercado, crescimento, imitação, etc. As rotinas incluem regras de decisão em todos os níveis, incluindo busca de novas técnicas de produção, que incluem regras quanto ao uso dos insumos e produtos, regras para investimentos, além de políticas relacionadas à PEBD. O resultado das práticas adotadas só será conhecido futuramente, quando o processo de concorrência entre as empresas determinar quais foram as estratégias vencedoras, através do desempenho que cada uma delas obteve. O sistema de mercado, por um processo de seleção, vai escolher as melhores práticas, que passam a ser difundidas, eliminando aquelas consideradas ineficientes. A busca constante por inovação, as rotinas traçadas pelas empresas e a seleção do mercado pelas melhores estratégicas são componentes fundamentais do processo de concorrência (NELSON; WINTER, 1982).

Para Dosi (1988), outro importante autor evolucionário, as firmas tendem a se diferenciar umas das outras, sendo que o mesmo processo ocorre entre regiões e países, gerando níveis de desempenho econômico assimétricos. Um fator fundamental que leva a essa diferenciação é o tipo de tecnologia adotada. Cada tecnologia possui características que imprimem maior ou menor dinamismo à economia. Quanto maior for o grau de conhecimento exigido para a adoção de uma dada tecnologia (cumulatividade) e sua capacidade de geração de inovação de produto e processo (oportunidade) e quanto mais se necessitar para o aprendizado do uso da tecnologia de relações interpessoais (caráter tácito e específico), maior será o poder de apropriação de lucros extraordinários (apropriabilidade) pela empresa, região ou país. Na atual fase do capitalismo, o paradigma tecnológico mais dinâmico é o das tecnologias de informação e comunicação (TICs). As regiões que 
possuem maior número de empresas inseridas nesse paradigma são as que mais têm crescido.

Dosi (1982) define paradigma tecnológico como certo padrão de resolução de problemas tecnológicos específicos, tendo como suporte os princípios de determinadas ciências naturais e as tecnologias de certos elementos materiais. Assim, por exemplo, a amplificação e comutação de sinais elétricos estariam relacionadas a um paradigma tecnológico, baseado no desenvolvimento da física do estado sólido e tendo como elemento fundamental o silício.

As formas concretas pelas quais cada paradigma tecnológico se desenvolve são denominadas trajetórias tecnológicas, que mostram o progresso da tecnologia no tempo. Cada paradigma pode conter várias trajetórias tecnológicas. A trajetória tecnológica é definida como uma atividade que resolve problemas tecnológicos dentro de um dado paradigma. Dada tecnologia se desenvolve, assim, ao longo de uma trajetória, segundo suas características específicas de cumulatividade, apropriabilidade, oportunidade e caráter tácito e específico. Tais características da tecnologia consistem em importantes fontes de geração de assimetrias entre firmas, indústrias e países. Em suma, para Dosi (1982, 1988), a adoção de determinadas tecnologias imprimem dinâmicas de crescimento distintas entre empresas, regiões e países.

A contribuição destacada de Pavitt (1984) a essa literatura é a taxonomia elaborada de todos os setores produtivos da economia, a partir de um estudo empírico, que procura agrupá-los segundo um conjunto de fatores relacionados, sobretudo, tecnológicos. Tal taxonomia permite entender o padrão de concorrência dos setores. Com esse intuito, os agrupamentos ficaram da seguinte forma: setores dominados por fornecedores; setores de produção intensiva, subdivididos em intensivos em escala e fornecedores especializados; e setores baseados em ciência (science based).

Primeiramente, os principais setores dominados pelos fornecedores são a agricultura, a construção civil, os serviços privados e a manufatura tradicional. As principais características desse grupo são: o tamanho relativamente pequeno das firmas inovadoras; as fontes da tecnologia de processo são os fornecedores, os serviços de pesquisa e extensão e os grandes usuários; os meios de apropriação derivam de propaganda, marcas e outros fatores não técnicos; e as trajetórias tecnológicas estão centradas em redução de custos.

Segundo, os setores intensivos em escala são de produtos intermediários e duráveis de consumo, incluindo automóveis. As características principais desses setores são: o tamanho das firmas inovadoras é grande; o desenvolvimento tecnológico provém da engenharia de produção, de fornecedores e de PEBD próprios; a apropriação é obtida através de segredos de processo e know-how, defasagens 
técnicas, patentes e aprendizado; e as tecnologias estão direcionadas para redução de custos e design de produto.

Terceiro, os fornecedores especializados compreendem as maquinarias e os instrumentos de precisão, caracterizados por: tamanho relativamente pequeno das empresas inovadoras; fontes de tecnologia provindas de projeto, de desenvolvimento e de usuários; usuários sensíveis ao desempenho do produto; apropriação derivada de know-how do projeto, conhecimento dos usuários e patentes; e trajetória tecnológica direcionada para o projeto do produto.

Por fim, as indústrias science based compreendem as indústrias eletrônica, elétrica e química. Suas principais características são: tamanho grande das firmas inovadoras; tecnologia derivada de $\mathrm{P} \mathcal{E} \mathrm{D}$ próprios e pesquisa pública; usuários que buscam melhor preço e melhoria do produto; fonte de apropriação constituída de know-how em PED, patentes, segredo e know-how de processos e aprendizado; e desenvolvimento tecnológico que visa redução de custos e melhoria do produto.

$\mathrm{Na}$ taxonomia de Pavitt (1984), embora procure incluir os setores primário e terciário, o foco recai, principalmente, sobre o setor industrial. Os serviços de telecomunicações, estudado nesse artigo, está conectado diretamente à indústria, tanto como fornecedora, quanto como compradora. Porém, como serviço, requer uma taxonomia própria.

Castellacci (2008) procura cobrir essa lacuna, afirmando que os serviços de infraestrutura e distributivos, dentre eles os de telecomunicações, demandam muitos equipamentos da indústria, exigindo constante inovação de seus fornecedores. Por sua vez, melhorias nesses serviços têm um efeito grande no sistema de inovação como um todo, reduzindo custos e gerando aumentos de produtividade na economia. Os serviços de telecomunicações também estão estreitamente conectados com outros serviços como os de softwares e PED. Em outras palavras, no paradigma das TICs, há uma interdependência e interações verticais entre indústria e serviços.

A fim de representar essas interações, Castellacci (2008) propõe uma nova taxonomia em que os segmentos produtivos seriam agrupados em quatro grandes grupos: a) fornecedores de conhecimento avançado; b) bens de produção em massa; c) serviços de infraestrutura de suporte; e d) bens e serviços pessoais. $\mathrm{O}$ autor, ainda, subdivide os três primeiros grupos em dois: aqueles que têm como suporte o novo paradigma tecnológico das TIC's e os que têm como base o paradigma fordista. Os bens e serviços pessoais, por sua vez, incorporam tecnologias de diferentes paradigmas.

Os serviços de telecomunicações, foco deste trabalho, se encontram no terceiro grupo, que, juntamente com as finanças, utilizam de forma intensa o novo paradigma tecnológico das TIC's. Quanto ao regime tecnológico, Castellacci (2008) classifica esse setor da seguinte forma: a) a oportunidade de investimento é 
de nível médio; b) a fonte externa de tecnologia consiste nos fornecedores e usuários; c) a apropriabilidade vem da capacidade das empresas de elaborar padrões, normas e design; e d) o tamanho da empresa é grande. Em relação às trajetórias tecnológicas, o setor é classificado da seguinte forma: a) em relação ao tipo de inovação, como sendo de processos mistos de inovação de serviço e organizacional; e b) em relação aos gastos e estratégias de inovação, como direcionado a PEBD, aquisição de software e treinamento.

Pela sua natureza, como mencionado, os serviços de telecomunicações estão diretamente relacionados com todos os demais grupos. Eles se encontram no início da cadeia vertical, uma vez que são mais ofertantes de intermediários do que de consumo final. Diferem do grupo denominado fornecedores de conhecimento avançado porque não têm a mesma capacidade de desenvolver internamente a tecnologia. Porém, dependem dos fornecedores de maquinarias, equipamentos e vários tipos de conhecimento avançado criados no sistema econômico como um todo. Por sua vez, quanto mais tecnologicamente desenvolvidos tornam-se os serviços de telecomunicações, maior será o efeito sobre a produtividade de todos os demais setores, gerando maior dinamismo à economia.

Em suma, a nova taxonomia de Castellacci (2008) permite uma melhor compreensão sobre as fontes de desenvolvimento dos serviços de telecomunicações e sobre sua capacidade de geração de dinamismo no sistema econômico.

Além das obras seminais e de seus refinamentos teóricos, um refinamento empírico da teoria evolucionária está contida na concepção do Manual de Oslo (1997), elaborado pela Organização para Cooperação Econômica e Desenvolvimento (OCDE), em que se define também como ocorre a inovação, tanto em termos de produto, quanto de processo. Entre seus vários objetivos, estabelece um padrão internacional para o conceito de inovação tecnológica por parte das empresas de qualquer segmento da economia e de qualquer país.

Segundo a Organização para Cooperação Econômica e Desenvolvimento (1997), quando as empresas realizam um processo inovativo, fazem parte de um complexo conjunto de atividades com múltiplos resultados, alguns dos quais podem reformular as fronteiras e a natureza da própria empresa. A grande questão desse processo inovativo está em decidir quais dessas atividades e desses resultados devem e podem ser mensurados.

O manual se concentra em duas categorias das cinco identificadas por Schumpeter (1982): produtos e processos novos e aprimorados, desde que exijam uma melhoria objetiva no desempenho de um determinado produto, não sendo considerados, por exemplo, mudanças nos produtos que sejam de caráter estético.

Nesse sentido, o presente estudo selecionou as variáveis segundo os critérios desse manual. 
Uma inovação tecnológica em produto e processo (TPP) é considerada implantada se tiver sido introduzido um novo produto no mercado (inovação de produto) ou utilizado um novo processo de produção (inovação de processo), envolvendo uma série de atividades científicas, tecnológicas, organizacionais, financeiras e comerciais. Uma empresa inovadora em TPP é, portanto, aquela que tenha implantado produtos ou processos tecnologicamente novos ou com substancial melhoria tecnológica durante o período em análise. Eles devem ser novos (ou substancialmente melhorados) para a empresa, e não, necessariamente, novos no mundo, podendo estar relacionados a bens ou serviços (ORGANIZAÇÃO PARA COOPERAÇÃO ECONÔMICA E DESENVOLVIMENTO, 1997).

Um produto tecnologicamente novo pode envolver tecnologias radicalmente novas, pode se basear na combinação de tecnologias existentes em novos usos ou pode ser derivado do uso de novo conhecimento. Já o produto tecnologicamente aprimorado (em termos de melhor desempenho ou menor custo) pode utilizar componentes ou materiais de desempenho melhores ou vários subsistemas técnicos integrados.

Tais produtos podem ter grandes e pequenos efeitos na empresa. Por exemplo, a substituição de metais por plásticos nos equipamentos de cozinha ou mobílias, que consiste na substituição por componentes de melhor desempenho, e a introdução de freios ABS ou outras melhorias de subsistemas em automóveis, que consiste em mudanças parciais em alguns subsistemas técnicos integrados.

A distinção entre um produto tecnologicamente novo e um produto tecnologicamente aprimorado pode apresentar dificuldades de distinção em alguns setores, especialmente no de serviços.

A inovação tecnológica de processo é a adoção de métodos de produção novos ou significativamente melhorados, incluindo métodos de entrega dos produtos. Tais métodos podem envolver mudanças no equipamento ou na organização da produção, ou uma combinação dessas mudanças, e podem derivar do uso de novos conhecimentos. Os métodos podem ter por objetivo produzir ou entregar produtos tecnologicamente novos ou aprimorados que não possam ser produzidos ou entregues com os métodos convencionais de produção ou pretender aumentar a produção ou eficiência na entrega de produtos existentes.

Em algumas indústrias de serviço, a distinção entre processo e produto pode ser nebulosa. Por exemplo, uma mudança de processo em telecomunicações para introdução de uma rede inteligente pode permitir a oferta ao mercado de um conjunto de novos produtos, tais como chamada em espera ou visualização da chamada (ORGANIZAÇÃO PARA COOPERAÇÃO ECONÔMICA E DESENVOLVIMENTO, 1997).

Segundo a Organização para Cooperação Econômica e Desenvolvimento (1997), as empresas do setor de serviços são as principais usuárias da inovação 
gerada nas indústrias manufatureiras e, portanto, desempenham um importante papel na geração do conhecimento.

Em muitos campos, a fronteira entre indústria e serviços como setores inovadores torna-se cada vez mais indistinta (o software, por exemplo, representa uma parcela cada vez mais significativa na maioria das inovações que são informadas como originadas na indústria). Nesse sentido, para separar corretamente as informações para o setor de serviços, o Manual de Oslo (1997) leva em consideração as seguintes características:

a) a inovação nas indústrias de serviço é distinta daquelas da indústria manufatureira. A inovação em serviços é frequentemente de natureza imaterial e, portanto, difícil de proteger. O setor de serviços tem um maior grau de ajuste às necessidades particulares de cada cliente. Há uma maior inter-relação entre o desenvolvimento de novos serviços e os processos que os produzem;

b) há diferenças no contexto estatístico. Existem programas estatísticos estabelecidos para os serviços de movimentação de mercadorias, inclusive de frete e transporte do comércio no atacado e no varejo. Isso significa que há robustas medidas da produção, do investimento, dos preços e da atividade financeira para essas indústrias, o que torna mais fácil distinguir as diferenças entre inovadores e não inovadores e fazer as inferências de políticas. Para as indústrias que não estão diretamente relacionadas com a movimentação de mercadorias, o pano de fundo para a aferição da inovação é menos claro e algumas dessas indústrias de serviços são economicamente significativas, além de serem instrumentos de mudanças tecnológicas e sociais. Dentre elas podem ser citadas as seguintes áreas: comunicações, finanças, seguros, imóveis, diversão e serviços de negócios;

c) as empresas da indústria de serviços tendem a ser menores do que as manufatureiras e menos concentradas. Isso tem implicações metodológicas para pesquisas por amostragem e para as estimativas da indústria;

d) nem todas as indústrias de serviço são iguais. Elas podem exigir competências distintas, organizar de formas diversas suas funções de produção e marketing, utilizar distintos níveis de tecnologia e atender diferentes mercados. Assim, podem apresentar diferentes inclinações para engajar-se no comércio internacional para inovar e, portanto, respondem de forma diversa às condições econômicas.

O Manual de Oslo (1997) tornou-se marco para os estudos a respeito da inovação tecnológica em todo o mundo e para os mais diversos setores de qualquer economia. Tal fato justifica-se pelo nível de detalhamento que apresenta no tocante à inovação e sua distinção entre produto e processo. Nesse sentido, o manual serviu de base para as Pintecs realizadas desde 2000 (de forma trienal), elaboradas 
pelo IBGE, sendo a de 2008 a última versão disponível. As pesquisas são realizadas com o objetivo de fornecer informações para a construção de indicadores setoriais, nacionais e regionais das atividades de inovação tecnológica das empresas brasileiras com dez ou mais pessoas ocupadas, tendo como universo de investigação as atividades das indústrias extrativas e de transformação e de serviços selecionados, tais como: edição, telecomunicações, informática e PËD.

Os detalhes a respeito das variáveis e seus tratamentos são elucidados na seguinte seção.

\section{Metodologia}

Esta seção mostra os métodos analíticos e a fonte e tratamento de dados utilizados no presente estudo. A técnica multivariada de análise fatorial foi utilizada na construção do Índice de Inovação.

\subsection{Análise Fatorial}

A construção do índice de capacidade de inovação (ICI) das empresas do setor de serviços de telecomunicações foi feita com base na análise fatorial por componentes principais. O modelo de análise fatorial é apresentado, genericamente, em forma matricial:

$$
X=\mu+\alpha f+\varepsilon
$$

em que $X=\left(X_{1}, X_{2}, X_{3}, X_{4}, X_{5}, X_{6}, X_{7}, X_{8}, X_{9}, X_{10} \text { e } X_{11}\right)^{\mathrm{t}}$ (variáveis definidas na Tabela 1 da seção fonte e tratamento de dados) é um vetor transposto de variáveis aleatórias observáveis; $f=\left(f_{1}, f_{2}, \ldots, f_{r}\right)^{t}$ é um vetor transposto $(r<p)$ de variáveis não observáveis ou fatores; $\alpha$ é uma matriz ( $p \times r$ ) de coeficientes fixos ou cargas fatoriais; e $\varepsilon=\varepsilon_{1}, \varepsilon_{2}, \ldots, \varepsilon_{p}$ é um vetor transposto de erros aleatórios.

A análise fatorial possui propriedades importantes: a primeira é que $\mathrm{E}(\varepsilon)=\mathrm{E}(f)=0$ e a segunda refere-se aos fatores que devem ser ortogonais. No presente estudo, foi utilizado o método Varimax de rotação ortogonal dos fatores. Tal metodologia será necessária porque nem sempre a estrutura inicial das estimativas das cargas fatoriais é definitiva e, além disso, o referido método melhora a interpretação dos fatores com as variáveis em estudo. ${ }^{4}$

4 Para mais detalhes sobre a análise fatorial, ver Corrar et al. (2009). 


\subsection{Construção do Índice de Capacidade de Inovação}

A propriedade de ortogonalidade dos escores fatoriais estimados foi utilizada para a elaboração do ICI. Entretanto, deve-se observar que a ortogonalidade, associada à matriz de fatores, não implica necessariamente ortogonalidade dos escores fatoriais, devendo-se testar se os escores fatoriais são ortogonais por meio da matriz de variância e covariância entre esses escores.

O índice parcial de inovação (IPI) pode ser estimado por meio da expressão 2:

$$
\mathrm{IPI}_{\mathrm{i}}=\left(\sum_{\mathrm{i}=1}^{\mathrm{n}} \mathrm{F}_{\mathrm{j}}^{2}\right)^{\frac{1}{2}} \operatorname{com} \mathrm{j}=1,2, \ldots, \mathrm{p}
$$

em que $\mathrm{IPI}_{i}$ é o índice parcial de inovação à i-ésima empresa do setor de serviços de telecomunicações e $F_{i j}$ é os escores fatoriais estimados, conforme o procedimento dos componentes principais.

Espera-se que os escores associados às empresas tenham distribuição simétrica em torno da média zero. Assim, metade apresenta sinais negativos e a outra metade, sinais positivos, de modo que as empresas com maiores ICI e competitividade parciais apresentarão escores fatoriais negativos. A fim de se evitar que altos escores fatoriais negativos elevem a magnitude dos índices associados a essas empresas, é conveniente inseri-los no primeiro quadrante, conforme a seguinte normalização:

$$
F_{i j}=\frac{\left(F-F_{\text {min }}\right)}{\left(F_{\max }-F_{\min }\right)}
$$

em que $F_{\text {min }}$ e $F_{\text {max }}$ são os valores mínimo e máximo observados para os escores fatoriais associados às empresas do setor de serviços de telecomunicações. Por meio desse procedimento, consegue-se alocar todos os escores fatoriais no intervalo fechado entre zero e um. O cálculo geométrico dos IPIs é mostrado na Figura 1. 
Figura 1 - Construção geométrica do IPI

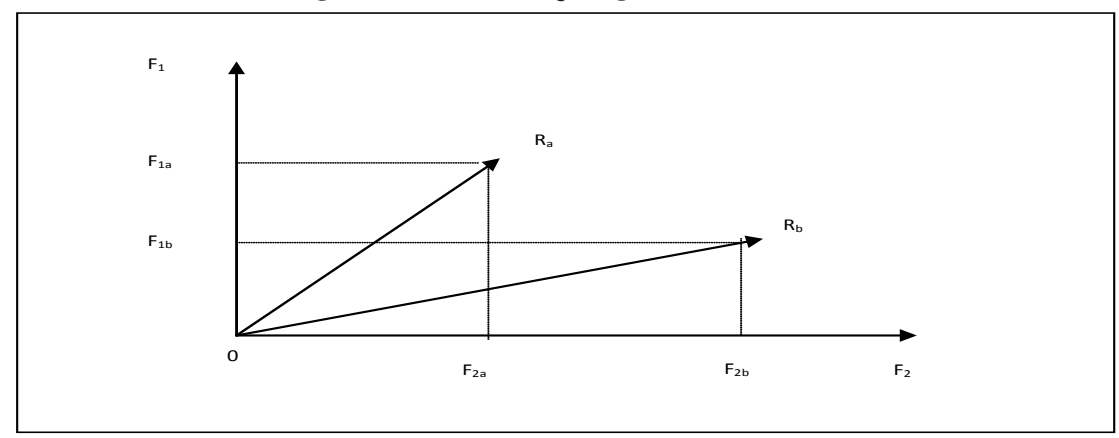

Fonte: Rossato (2006).

Observa-se, na Figura 1, que, associados à empresa $A$, estão os escores fatoriais $F_{1 a}$ e $F_{2 a}$. $O$ vetor $R_{a}$ é a resultante associada a esses escores fatoriais ortogonais, assim definidos:

$$
R_{a}=\left(F_{1 a}^{2}+F_{2 a}^{2}\right)^{1 / 2}
$$

Um procedimento semelhante é utilizado para a empresa $B$. $O$ tamanho da resultante $R_{a}$ ou $R_{b}$ determina a magnitude do IPI associado às empresas $A$ e $B$, respectivamente. Ressalte-se que o IPI, definido dessa forma, é necessário para fazer o ranking das empresas do setor de serviços de telecomunicações quanto ao nível de capacidade de inovação. O IPI não pode ser utilizado para estimar o percentual de capacidade de inovação de cada uma das empresas. Para esse cálculo, utiliza-se o ICI, cuja construção, associada à i-ésima empresa, define-se conforme a formulação a seguir.

$$
I C I_{i}=\left(\sum_{i=1}^{n} P_{j} X_{i}\right)
$$

em que os pesos $P_{j}$ são estimados por mínimos quadrados restritos, o $I P I_{i}$ é a variável dependente e as variáveis explicativas são os indicadores utilizados para a construção do mesmo. A expressão 6 resume o exposto: ${ }^{5}$

$$
\begin{aligned}
& I P I=\beta_{0}+\beta_{1} V Q+\beta_{2} X_{5}+\beta_{3} X_{6}+\beta_{4} X_{7}+\beta_{5} X_{8}+\beta_{6} X_{9}+\beta_{7} X_{10}+\beta_{8} X_{11}+\mu \\
& \text { s.a. } \\
& \beta_{1}+\beta_{2}+\beta_{3}+\beta_{4}+\beta_{5}+\beta_{6}+\beta_{7}+\beta_{8}=1
\end{aligned}
$$

5 Para mais informações, ver Greene (2000) e Gujarati (2006). 


\subsection{Fonte e Tratamento dos Dados}

Não há consenso na literatura econômica quanto à utilização de variáveis que possam definir a capacidade de inovação, ficando o pesquisador apto a selecionar os atributos (variáveis) que permitam melhor realizar a análise do espaço geográfico em estudo (SOARES et al., 1999).

O presente trabalho abordou o IED e o desenvolvimento tecnológico não de maneira agregada, mas de modo mais detalhado possível, utilizando-se os microdados coletados

Como o propósito é apurar a inovação tecnológica do setor, realizou-se uma análise a priori da Pintec para se selecionar quais seriam as variáveis que iriam compor o índice. Para o setor de serviços de telecomunicações, estão disponíveis dados a partir da Pintec de 2005. Para o ano de 2008, a Pintec ainda não estava disponível para consulta.

A escolha da Pintec justifica-se por dois principais motivos: o primeiro, por estar em conformidade com o que a literatura neoschumpteriana preconiza como variáveis relevantes para se analisar os efeitos da inovação tecnológica no desenvolvimento econômico. O segundo, pelo fato de a Pintec ser uma base de dados detalhada sobre inovação tecnológica de empresas no Brasil. O uso das informações em nível de empresa mostra a qualidade e a confiabilidade das análises realizadas para o setor.

A Pintec possui um questionário composto por 197 perguntas sobre inovação tecnológica industrial e, mais recentemente, sobre serviços. Dessa forma, foi necessário realizar uma análise de todas as perguntas para se apurar quais seriam as que melhor se adequariam ao índice proposto.

Uma característica do questionário é ser composto por um grande número de variáveis qualitativas e poucas variáveis quantitativas. Do universo de 197 questões, apenas 54 não são de natureza qualitativa. No presente trabalho, o cálculo do índice de inovatividade proposto requer a utilização da análise fatorial. Entretanto, como as questões da Pintec são, em sua maioria, qualitativas, optou-se por uma redução do número dessas variáveis (duas), de forma a minimizar problemas estatísticos.

É importante ressaltar que a construção do ICI envolve um conhecimento a priori de quais seriam os níveis ideais de inovação associados aos indicadores que são utilizados para a sua construção. Devido às variações que podem ocorrer conforme quem estiver fazendo a análise, tal tarefa não é realizada facilmente justamente por essa característica subjetiva.

Para se evitar tal subjetividade, fez-se preliminarmente uma análise minuciosa do questionário com o objetivo de se identificar a sua uniformidade de informações. 
Constatou-se que muitas das questões contidas na Pintec não eram respondidas pelas empresas. Com isso, essas questões não foram utilizadas na construção do ICI.

Foi necessário também avaliar as empresas que se propuseram a responder ao questionário, e constatou-se que não havia uma uniformidade entre as que compunham o setor. As empresas que responderam a um número muito baixo de questões não foram consideradas no cômputo do índice, reduzindo-se, assim, a amostra de 133 empresas (o número total de empresas que responderam o questionário) para 87 (37 estrangeiras e 50 nacionais) que efetivamente responderam as questões relevantes para a análise.

As variáveis utilizadas no estudo estão descritas na Tabela 1.

Tabela 1 - Denominação das questões da Pintec para o cômputo do ICI

\begin{tabular}{|c|c|}
\hline Questões da Pintec & Denominação \\
\hline $\begin{array}{l}\text { A empresa introduziu (no período de } 2003 \text { a 2005) produto } \\
\text { (bem ou serviço) tecnologicamente novo ou significativamen- } \\
\text { te aperfeiçoado para a empresa, mas já existente no mercado } \\
\text { nacional? (questão 10) }\end{array}$ & $\mathrm{X} 1$ \\
\hline $\begin{array}{l}\text { A empresa introduziu (no período de } 2003 \text { a 2005) produto } \\
\text { (bem ou serviço) tecnologicamente novo ou significativamen- } \\
\text { te aperfeiçoado para o mercado nacional? (questão 11) }\end{array}$ & $\mathrm{X} 2$ \\
\hline $\begin{array}{l}\text { A empresa introduziu processo (no período de } 2003 \text { a 2005) } \\
\text { tecnologicamente novo ou significativamente aperfeiçoado para } \\
\text { a empresa, mas já existente no setor no Brasil? (questão 16) }\end{array}$ & $\mathrm{X} 3$ \\
\hline $\begin{array}{l}\text { A empresa introduziu processo (no período de } 2003 \text { a 2005) } \\
\text { tecnologicamente novo ou significativamente aperfeiçoado } \\
\text { para o setor no Brasil? (questão 17) }\end{array}$ & $\mathrm{X} 4$ \\
\hline $\begin{array}{l}\text { Valor gasto com atividades relacionadas à pesquisa e desenvol- } \\
\text { vimento desenvolvidas internamente na empresa (questão 31) }\end{array}$ & $\mathrm{X} 5$ \\
\hline $\begin{array}{l}\text { Valor gasto com aquisição de outros conhecimentos externos, } \\
\text { exclusive software (questão 33) }\end{array}$ & $\mathrm{X} 6$ \\
\hline $\begin{array}{l}\text { Valor gasto com aquisição de máquinas e equipamentos } \\
\text { (questão 34) }\end{array}$ & $\mathrm{X} 7$ \\
\hline Valor gasto em treinamento (questão 35) & $\mathrm{X} 8$ \\
\hline $\begin{array}{l}\text { Valor gasto com introdução das inovações tecnológicas no } \\
\text { mercado (questão 36) }\end{array}$ & X9 \\
\hline $\begin{array}{l}\text { Valor gasto com projeto industrial e outras preparações técni- } \\
\text { cas para a produção e distribuição (questão 37) }\end{array}$ & $\mathrm{X} 10$ \\
\hline $\begin{array}{l}\text { A empresa dispunha de alguma patente em vigor no final de } \\
\text { 2005? (questão 173) }\end{array}$ & $\mathrm{X} 11$ \\
\hline
\end{tabular}

Fonte: Elaboração própria a partir de IBGE (2005). 
As variáveis X1, X2, X3 e X4 são categóricas, logo a empresa entrevistada respondeu apenas "sim" ou "não" para cada uma das perguntas. Como o objetivo do presente estudo concentra-se no cálculo para mensuração de atividade tecnológica após a privatização no setor de telecomunicações, e como já posto inicialmente, variáveis categóricas (ou binárias como é o caso das referidas perguntas) não são estatisticamente confiáveis para apuração de índice, então realizou-se também o tratamento descrito na Tabela 2 para as variáveis com essa característica.

Tabela 2 - Valor por combinação de respostas

\begin{tabular}{ccccc|ccccc}
\hline VQ & X1 & X2 & $\mathbf{X 3}$ & $\mathbf{X 4}$ & VQ & X1 & X2 & X3 & X4 \\
\hline $\mathbf{1 6}$ & $\operatorname{sim}$ & $\operatorname{sim}$ & $\operatorname{sim}$ & $\operatorname{sim}$ & $\mathbf{8}$ & não & sim & não & não \\
$\mathbf{1 5}$ & $\operatorname{sim}$ & $\operatorname{sim}$ & não & $\operatorname{sim}$ & $\mathbf{7}$ & $\operatorname{sim}$ & não & não & sim \\
$\mathbf{1 4}$ & $\operatorname{sim}$ & $\operatorname{sim}$ & $\operatorname{sim}$ & não & $\mathbf{6}$ & não & não & sim & sim \\
$\mathbf{1 3}$ & não & $\operatorname{sim}$ & $\operatorname{sim}$ & $\operatorname{sim}$ & $\mathbf{5}$ & não & não & não & sim \\
$\mathbf{1 2}$ & $\operatorname{sim}$ & não & $\operatorname{sim}$ & $\operatorname{sim}$ & $\mathbf{4}$ & $\operatorname{sim}$ & não & sim & não \\
$\mathbf{1 1}$ & não & $\operatorname{sim}$ & não & $\operatorname{sim}$ & $\mathbf{3}$ & $\operatorname{sim}$ & não & não & não \\
$\mathbf{1 0}$ & não & $\operatorname{sim}$ & $\operatorname{sim}$ & não & $\mathbf{2}$ & não & não & sim & não \\
$\mathbf{9}$ & sim & $\operatorname{sim}$ & não & não & $\mathbf{1}$ & não & não & não & não \\
\hline
\end{tabular}

Fonte: Elaboração própria a partir de IBGE (2005).

Percebe-se que, entre as quatro perguntas, a diferença está entre a inovação de processo ou a inovação de produto e se o processo ou o produto é novo para a empresa ou para o mercado interno. Portanto, há a possibilidade de a empresa entrevistada responder ou afirmativamente para ambos, ou negativamente para ambos, ou de forma alternada. Desse modo, com o objetivo de dirimir o uso de variáveis categóricas, mas sem cometer o erro de eliminar variáveis extremamente importantes para o objetivo do estudo, foram mapeadas todas as possibilidades de respostas para as quatro questões e, em seguida, foi elaborado um ranking para cada combinação de respostas.

Denominou-se a variável qualitativa (VQ) como a variável que combina essas respostas e que mede qual empresa foi mais intensiva em inovação, tanto por processo, quanto por produto, conforme a Tabela 2.

De acordo com cada combinação possível, atribuiu-se um valor quantitativo para cada combinação, ou seja, se a empresa respondeu "sim" para todas as questões, essa combinação tem um maior peso para o índice se comparado com a empresa que respondeu "não" para todas as questões. Portanto, sendo um conjunto de questões em que cada uma pode assumir até dois valores, tem-se um total de 16 possibilidades de respostas, de modo que o maior peso é dado àquele que apresentar valor 16 e o menor peso àquele que apresentar valor um. 
Como o setor de telecomunicações possui crescente demanda por aparelhos de comunicação com as mais variadas características (telefonia fixa, móvel, imagem, voz, texto, entre outros), considerou-se que a inovação por produto tem um maior peso, vis-à-vis à inovação por processo. Entretanto, segundo Patel e Vega (1999), a inovação por processo também ocorre em setores intensivos em alta tecnologia, tais como: computação, farmacêutico, imagem e som, materiais e telecomunicação.

No que diz respeito às variáveis de dispêndio (X5, X6, X7, X8, X9 e X10), não houve a necessidade de se realizar nenhum tratamento com relação à inflação ou paridade cambial com alguma moeda de referência, pois os valores são referentes ao ano de 2005 e todos em moeda nacional.

Em vez de se utilizar as variáveis em valor absoluto, optou-se por utilizá-las como uma proporção em relação à receita líquida de cada empresa. Desse modo, as variáveis utilizadas captam o montante que cada empresa dispõe de seu faturamento em prol de ganhos futuros que possam ser auferidos com investimento em inovação tecnológica.

A variável X11 foi tratada como variável dummy. Embora apresentando tal característica, sua permanência no estudo foi necessária pela importância teórica ao problema. As patentes requeridas indicam tanto a importância da inovação tecnológica realizada, quanto a importância que cada empresa atribui ao seu processo inovativo.

\section{Resultados}

A primeira fase da pesquisa para determinar o nível de inovação das empresas do setor de serviços de telecomunicação brasileiro consistiu na construção do índice de inovatividade para cada empresa do setor.

Para a construção desse índice, inicialmente as variáveis selecionadas foram reduzidas em fatores, utilizando-se o método de análise fatorial. Os testes indicaram que a amostra apresentou-se adequada à análise fatorial, ${ }^{6}$ que foi realizada pelo método de componentes principais. As variáveis foram reduzidas a quatro fatores com raízes características maiores que um, que contribuem com $68 \%$ da variância total dos indicadores utilizados (ver Tabela 3).

$6 \quad$ Foram realizados os testes de esfericidade de Bartlett e o teste de Kaiser-Meyer-Olkin. O teste de Bartlett atingiu valor igual a 673,84 , significativo a $1 \%$ de probabilidade, o que permite rejeitar a hipótese nula de que a matriz de correlação é uma matriz identidade, isto é, não existe correlação entre as variáveis. Para o teste de Kaiser-Meyer-Olkin, o valor obtido foi 0,747 , indicando que a amostra é adequada à realização da análise fatorial. 
Tabela 3 - Autovalores da matriz de correlação e variância explicadas na construção do ICI para as empresas do setor de serviços de telecomunicações

\begin{tabular}{ccccc}
\hline & Autovalores & Diferença & Proporção & Valor acumulado \\
\hline F1 & 8,765005 & 5,864252 & 0,3652 & 0,3652 \\
F2 & 2,900754 & 0,354617 & 0,1209 & 0,4861 \\
F3 & 2,546136 & 0,559145 & 0,1061 & 0,5922 \\
F4 & 1,986992 & & 0,0828 & 0,675 \\
\hline
\end{tabular}

Fonte: Elaboração própria.

Após a obtenção dos fatores e das cargas fatoriais, foram estimados os escores e, a partir destes, calculado o índice parcial de inovatividade. Entretanto, esse índice parcial não possibilita a ordenação das empresas em termos de quem é mais inovadora. Para se obter esse ranking, foi calculado o índice total de inovatividade, regredindo-se o índice parcial como variável dependente e as variáveis selecionadas na análise como independentes. O método utilizado para a estimação foi o de mínimos quadrados restritos.

Os resultados são ilustrados na Tabela 4 e expressam os pesos para cada variável quando da composição do índice de capacidade de inovação.

Observou-se que todas as variáveis obtiveram valor positivo e foram significativas a 1\%, exceto a variável X8 (valor gasto em treinamento), que se apresentou não significativa. Esse fato era esperado, já que sua participação no percentual de gasto em relação à receita líquida de vendas das empresas foi, em 2005, de apenas 0,02\% (IBGE, 2005). 
Tabela 4 - Resultados dos mínimos quadrados restritos para o peso das variáveis

\begin{tabular}{c|c|c|c|c}
\hline & Parâmetro estimado & Erro padrão & Valor t & Pr $>|\mathbf{t}|$ \\
\hline $\mathbf{X 5}$ & 0,609302 & 0,040957 & 6,541227 & 0,0000 \\
$\mathbf{X 6}$ & 0,267911 & 0,179274 & 8,976759 & 0,0000 \\
$\mathbf{X 7}$ & 2,330975 & 0,049692 & 6,660570 & 0,0000 \\
$\mathbf{X 8}$ & $-0,066818$ & 0,387412 & $-0,172473$ & 0,8635 \\
$\mathbf{X 9}$ & 1,498494 & 0,149731 & 3,329272 & 0,0013 \\
$\mathbf{X 1 0}$ & 0,427526 & 0,284669 & 8,527543 & 0,0000 \\
$\mathbf{X 1 1}$ & 0,219233 & 0,021118 & 10,38138 & 0,0000 \\
VQ & 0,016494 & 0,000884 & 18,65895 & 0,0000 \\
\hline
\end{tabular}

Fonte: Elaboração própria.

Nota: X5 é o valor gasto com atividades relacionadas à PẺD desenvolvidas internamente na empresa; X6 é o valor gasto com aquisição de outros conhecimentos externos, exclusive software; X7 é o valor gasto com aquisição de máquinas e equipamentos; X8 é o valor gasto em treinamento; X9 é o valor gasto com introdução das inovações tecnológicas no mercado; X10 é o valor gasto com projeto industrial e outras preparações técnicas para a produção e distribuição; e X11 é a empresa dispunha de alguma patente em vigor no final de 2005.

A partir da Tabela 4, observa-se que as variáveis X7 (valor gasto com aquisição de máquinas e equipamentos) e X9 (valor gasto com a introdução das inovações tecnológicas no mercado) foram as que geraram maior efeito no cálculo do índice. Em adição, X5 (valor gasto com atividades relacionadas à PËD desenvolvidas internamente na empresa), X10 (valor gasto com projeto industrial e outras preparações técnicas para a produção e distribuição), X6 (valor gasto com aquisição de outros conhecimentos externos, exclusive software) e X11 (existência de alguma patente em vigor no final de 2005) também foram importantes no cálculo do índice, assumindo a terceira, quarta, quinta e sexta posição no ranking das variáveis selecionadas, respectivamente. A variável VQ, que seria uma proxy para o esforço inovativo da empresa, foi a que apresentou menor coeficiente no índice proposto.

De acordo com dados da Pintec (IBGE, 2005), no setor de telecomunicações, as atividades de aquisição de máquinas e equipamentos possuem grande participação no percentual de gasto relativamente ao total da receita líquida de vendas das empresas. Após a aquisição de máquinas e equipamentos $(0,76 \%)$, a outra variável mais importante é a introdução das inovações no mercado $(0,72 \%)$ e as atividades internas de PEB (0,52\%). Esses resultados mostram que a ordenação do modelo está de acordo com a realidade observada.

A partir do cálculo dos pesos, pode-se construir o ICI. Conforme demonstra a Figura 2, de maneira geral as empresas não tiveram valor elevado para esse ín- 
dice. A maior parte das empresas, 49\%, obteve valor entre 0,20 e 0,40. Em adição, apenas $6 \%$ das empresas alcançaram valores superiores a 0,80.

Figura 2 - Frequência do ICI para as empresas analisadas

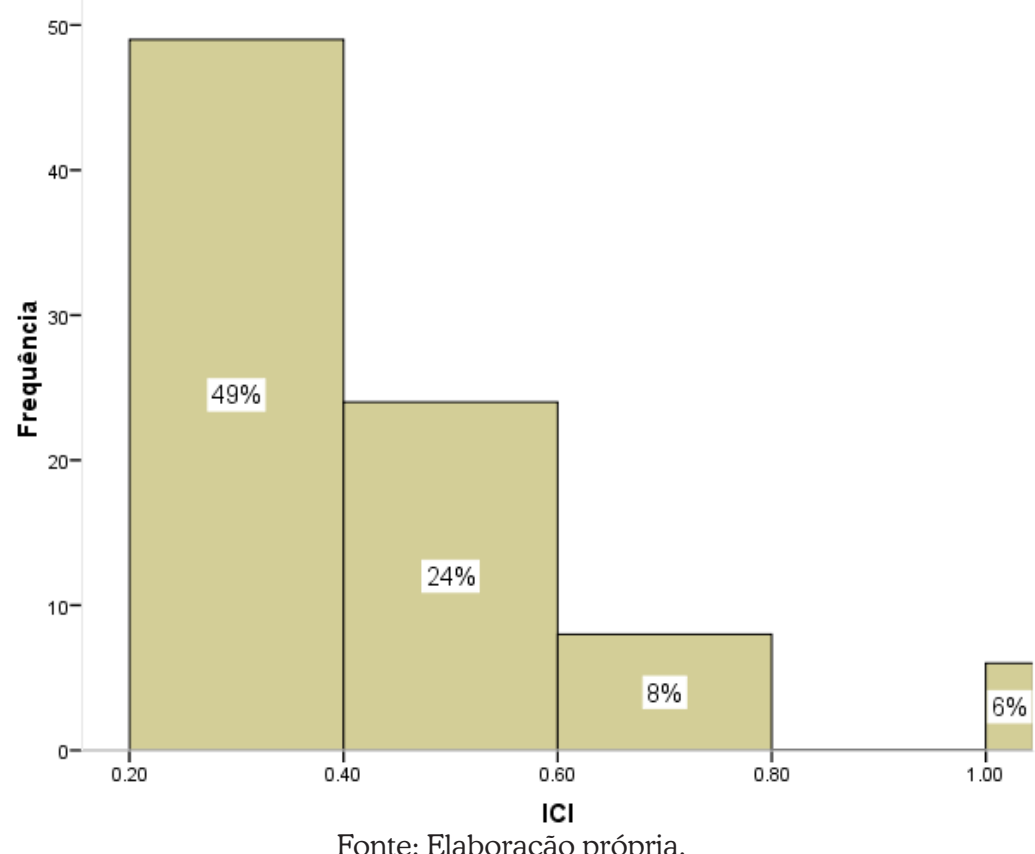

Fonte: Elaboração própria.

No que se refere ao tipo de empresa, nacional ou estrangeira, o menor índice foi observado para uma empresa nacional $(0,27)$. Em termos médios, não houve grande diferença entre os índices dos dois tipos de empresas: 0,4265 e 0,4196 para empresas estrangeiras e nacionais, respectivamente, indicando um patamar muito baixo de capacidade de inovação para um setor extremamente dinâmico e necessário para o desenvolvimento de outros setores da economia. A Tabela 5 ilustra os resultados expostos anteriormente.

Tabela 5 - Análise descritiva do ICI

\begin{tabular}{c|c|c|c}
\hline & Máximo & Mínimo & Média \\
\hline Nacionais & 1 & 0,2357 & 0,4196 \\
Estrangeiras & 1 & 0,2687 & 0,4265 \\
\hline
\end{tabular}

Fonte: Elaboração própria.

A Figura 3 evidencia a grande semelhança do valor do ICI para os dois tipos de empresas analisadas. Observa-se que, tanto para valores menores, quanto para 
valores próximos de um, visualmente não se consegue diferenciar as empresas nacionais e estrangeiras em termos de comportamento inovador.

Figura 3 - ICI para as empresas nacionais e estrangeiras

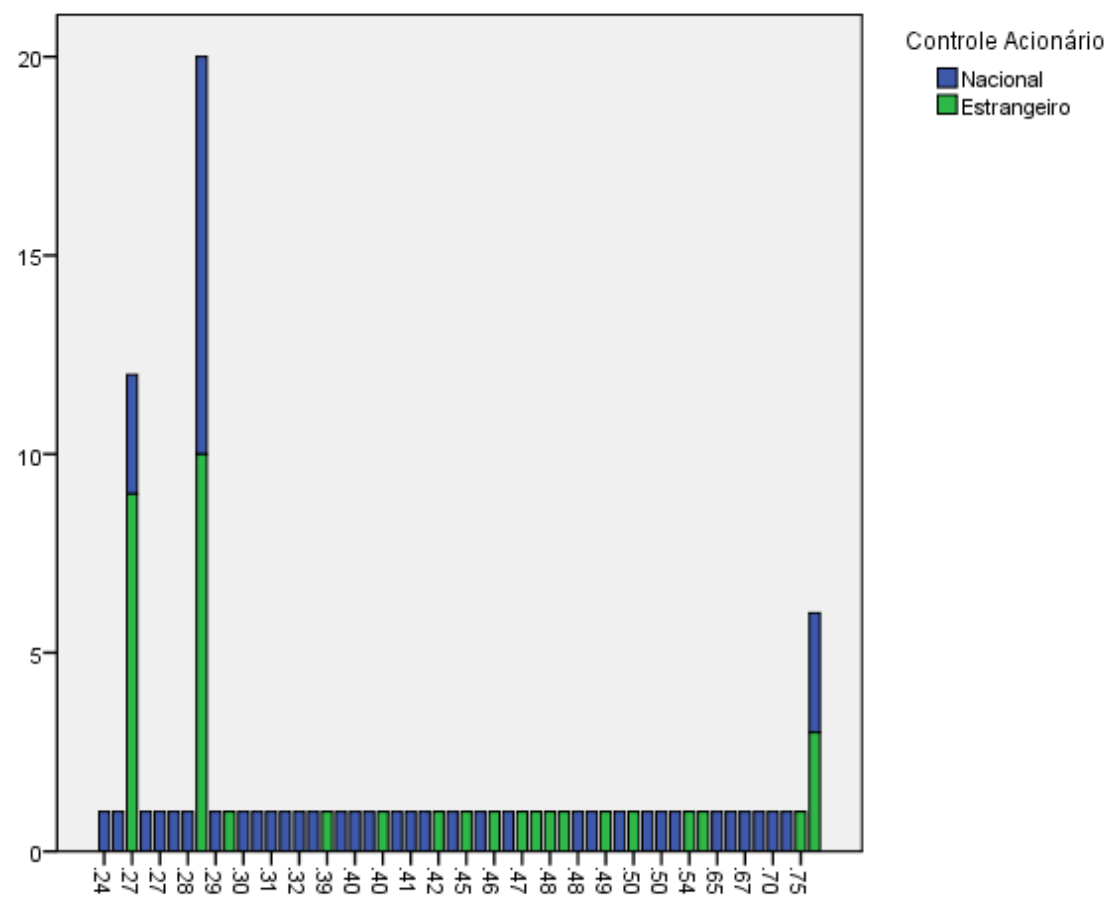

ICI

Fonte: Elaboração própria.

Em adição, estabeleceu-se uma relação entre o índice de capacidade de inovação e o indicador VQ que engloba variáveis representativas do resultado do sucesso inovativo (ver Figuras 4 e 5). As Figuras 4 e 5 mostram, respectivamente, a relação entre VQ e ICI para as empresas estrangeiras e nacionais. Observa-se que a tendência obtida para os dois tipos de empresas apresenta rendimentos decrescentes para o esforço inovativo. 
Figura 4 - Relação entre a variável VQ e o ICI para as empresas estrangeiras

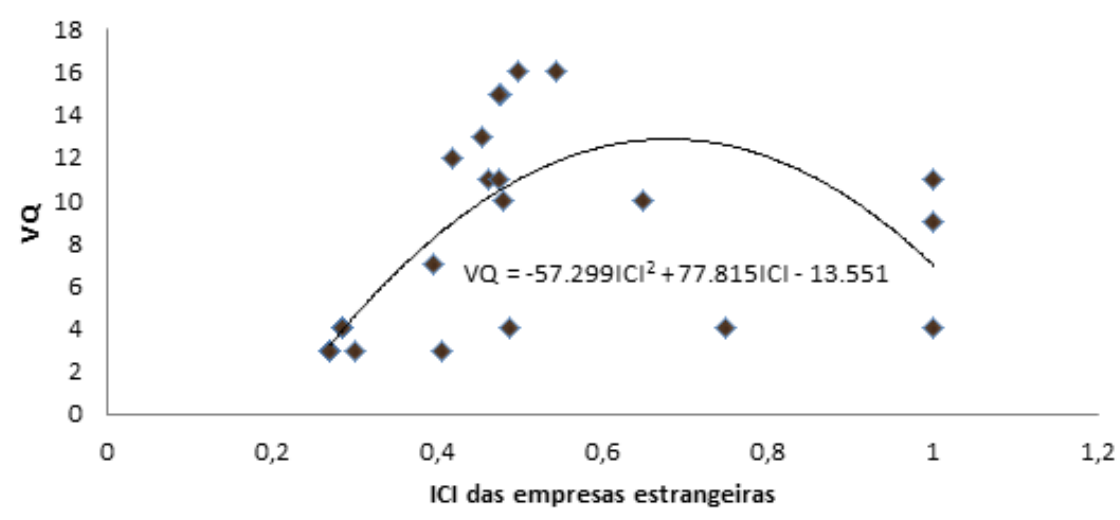

Fonte: Elaboração própria.

Figura 5 - Relação entre a variável VQ e o ICI para as empresas nacionais

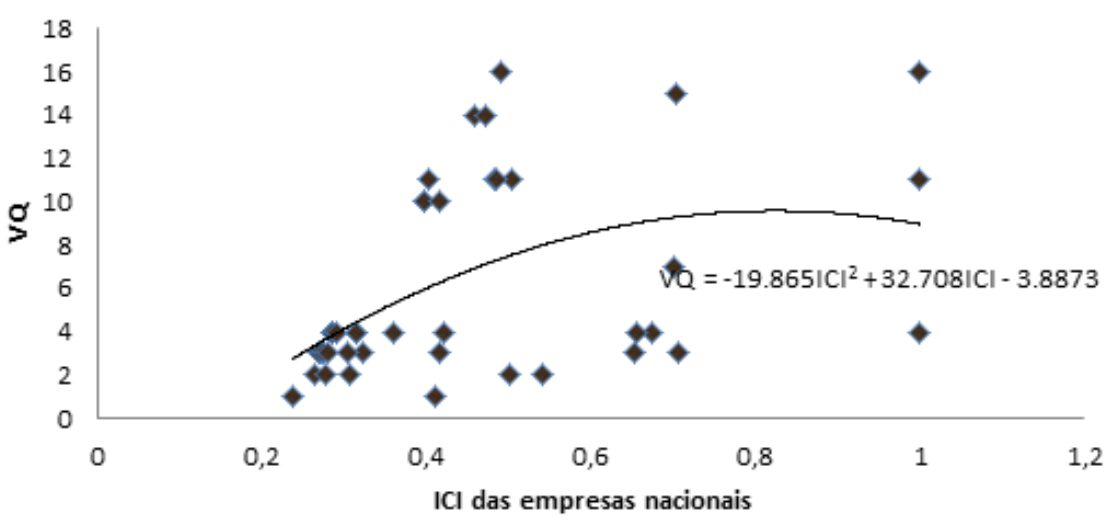

Fonte: Elaboração própria.

À medida que se aumenta o ICI, o sucesso inovativo, definido pelo nível de penetração ou alcance da inovação, $\mathrm{VQ}$, não cresce na mesma proporção, seja para as empresas estrangeiras ou nacionais. A partir de determinado nível de esforço, ocorre uma tendência de queda do sucesso inovativo. Isso acontece, primeiro, porque existem rendimentos decrescentes do esforço inovativo, o que implica esgotamento das oportunidades tecnológicas (conceito que pode ser entendido como quantidade de sucesso inovativo dado o montante de gastos com PED e outros gastos inovativos) colocadas pelo ambiente local (universidades, laboratórios públicos de pesquisa, spillover); segundo, porque firmas que extrapolam 
determinado patamar de esforço inovativo podem estar desenvolvendo projetos nas áreas de fronteira tecnológica, cujos sucessos não são imediatos.

De modo geral, observa-se que não existe grande diferença entre os ICIs das empresas nacionais e estrangeiras no setor de serviços de telecomunicações. Além disso, as médias obtidas para as empresas ficaram em torno de $40 \%$, valor considerado baixo para um setor que deve ter comportamento dinâmico (IBGE, 2005).

\section{Considerações Finais}

A privatização do setor de telecomunicações foi positiva para a economia no sentido de desenvolver a competitividade para o setor e trazer fluxos de capitais externos para o país, o que desenvolveu a concorrência em um setor totalmente dominado pelo Estado. Tal concorrência facilitou que os consumidores brasileiros (firmas e famílias) passassem a ter acesso a produtos e processos tecnológicos ainda desconhecidos no âmbito nacional.

O presente estudo buscou analisar o efeito da entrada de IED, em termos de inovação tecnológica, no setor de serviços de telecomunicação brasileiro. Com base nos resultados obtidos, pôde-se concluir que as empresas estrangeiras apresentaram uma pequena vantagem no processo inovativo, tanto em produto, quanto em processo, e a proximidade dos índices entre as empresas nacionais e estrangeiras impossibilitou afirmar, com veemência, que as estrangeiras foram tecnologicamente mais eficientes.

É importante salientar, ainda, que as empresas, tanto nacionais, quanto multinacionais tiveram retornos decrescentes ao esforço inovativo. As duas possíveis razões para isso estão na tendência de queda do sucesso inovativo a partir de determinado nível de esforço e nos sucessos mais lentos para firmas que desenvolvem projetos nas áreas de fronteira. Em seguida, observou-se que os valores médios do ICI foram baixos, tanto para as empresas nacionais, quanto para as estrangeiras. Esse resultado reforça a ideia de que o fluxo de IED direcionado para a economia brasileira veio para aproveitar mercado - market seeking, na denominação de Dunning (1981, 1988, 1999) - sem o estímulo e condições necessárias para realizar investimentos no desenvolvimento tecnológico local. Desse modo, o setor de serviços de telecomunicação parece ter seguido o mesmo comportamento de outros setores da economia em processo de fusão e aquisição. Tais resultados sugerem, primeiramente, que a atualização tecnológica do setor, como indicado, é fruto basicamente da aquisição de máquinas e equipamentos, sendo poucas inovações geradas internamente. Segundo, que não há esforço inovativo nem para adaptação de inovações geradas externamente, o que é bastante preocupante, dado que atualmente são necessários gastos em PẺD significativos para apenas imitar. 
Nesse contexto, surgem discussões importantes a respeito de quão desejável é o capital estrangeiro para a economia anfitriã, principalmente se esta não possui uma capacidade de absorção adequada para esse tipo de investimento, pois as multinacionais, na maioria das vezes, aproveitam tecnologia existente em suas matrizes. Conforme os preceitos teóricos que norteiam o processo inovativo por parte dessas empresas, nunca houve, no Brasil, características inerentes às empresas de telecomunicações privatizadas para que o país fosse centralizador de inovação tecnológica no setor. Tal fato reforça a ideia de que, apesar do mercado brasileiro ser atrativo para a entrada de capital estrangeiro, o governo não tem implementado políticas que mudem o foco desses investimentos tanto das empresas estrangeiras quanto das nacionais. A absorção de tecnologia já existente é desejável, mas um segundo passo seria capacitar os agentes a avançar no processo inovativo, a fim de que o país não fosse atrativo apenas do ponto de vista do mercado. Dessa forma, é natural que as multinacionais tivessem como foco, até o presente momento, apenas os ativos e o mercado brasileiro.

\section{Referências}

BAIN, J. S. Economies of scale, concentration, and the condition of entry in twenty manufacturing industries. The American Economics Review, v. 44, n. 1, p. 15-39, 1954.

CANTWELL, J. Technological innovation and multinational corporations. Oxford: Basil Blackwell, 1989.

CASTELLACCI, F. Technological paradigm, regimes and trajectories: manufacturing and services industries in a new taxonomy of sectoral patterns of innovation. Research Policy, v. 37, n. 6/7, p. 978-994, 2008.

CORRAR, L. J. PAULO, E.; DIAS FILHO, J. M. Análise multivariada para os cursos de administração, ciências contábeis e economia. São Paulo: Atlas, 2009.

CUILENBURG, J. V.; SLAA. P. Competition and innovation in telecommunications: an empirical analysis of innovative telecommunications in the public interest. Telecommunications Policy, v. 19, n. 8, p. 647-663, 1995.

DOSI, G. Institutions and markets in a dynamic world. The Manchester School, v. 56, n. 2, p. 119-146, 1988.

. Technological paradigm and technological trajectories: a suggested interpretation of the determinants and directions of technical change. Research Policy, v. 11, n. 3, p. 147-162, 1982.

DUNNING, J. H. Globalization and the theory of MNE activity. In: HOOD, N.; YOUNG, S. (Eds.). The globalization of multinational enterprise. London: Macmillan, 1999. 1981.

. International production and the multinational enterprise. London: G. Allen $\mathcal{E}$ Unwin, 
DUNNING, J. H. The eclectic paradigm of international production: a restatement and some possible extensions. Journal of international business studies, v. 19, n. 1, p.1-31, 1988.

DUNNING, J. H; RUGMAN, A. M. The influence of Hymer's dissertation on the theory of foreign direct investment. The American Economic Review, v. 75, n. 2, p. 228-232, 1985.

Reappraising the eclectic paradigm in the age of alliance capitalism. Journal of International Business Studies, 26, 461-491, 1995.

. The eclectic paradigm as an envelope for economic and business theories of MNE activity. International Business Review, v. 9, n. 1, p. 163-190, 2000.

. The eclectic (OLI) paradigm of international production: past, present and future. Journal of the Economics of Business, v. 8, n. 2, p. 173-190, 2001.

Perspectives on international business research: a professional autobiography fifty years researching and teaching international business. Journal of International Business Studies, v. 33, n. 4, p 817-835, 2002.

Towards a new paradigm of development: implications for the determinants of international business. Transnational Corporations, v. 15, n. 1, p. 173-227, 2006.

Institutional reform, FDI and the location competitiveness of European transition economies, in Dunning, J.H. and Gugler, P. (eds.), Foreign direct investment, location and competitiveness, Elsevier, Oxford, pp. 175-201, 2008

GALINA, S. V. R.; PLONSKI, G. A. Inovação no setor de telecomunicações no Brasil. Revista Brasileira de Inovação, v. 4, n. 1, p. 129-155, 2005.

GREENE, W. Econometric analysis. 5. ed. Englewood Cliffs: Prentice Hall, 2000.

GUJARATI, D. Econometria básica. 4. ed. Rio de Janeiro: Campus, 2006.

HYMER, S. H. The international operations of national firms: a study of direct foreign investment. Cambridge: MIT Press, 1976.

HYMER, S. H. The international operations of national firms: a study of direct foreign investment. 1960. 198 f. Thesis (Ph.D). Massachusetts Institute of Technology, 1960.

ISLAM, T.; FIEBIG, D. G.; MEADE, N. Modelling multinational telecommunications demand with limited data. International Journal of Forecasting, v. 18, n. 4, p. 605-624, 2002

LI, W.; XU, L. C. The impact of privatization and competition in the telecommunications sector around the world. Journal of Law and Economics, v. 47, n. 2, p. 395-430, Oct. 2004.

MADDEN, G. G.; SAVAGE, S. J. Telecommunications productivity, catch-up and innovation. Telecommunications Policy, v. 23, n. 1, p. 65-81, 1999.

NARULA, R.; ZANFEI, A. Globalization of innovation: the role of multinational enterprises. In: FAGERBERG, J.; MOWERY, D. C. The oxford handbook of innovation. Oxford: Oxford University Press. 2004. 
NELSON, R.; WINTER, S. An evolutionary theory of economic change. Cambridge: Belknap Press, 1982. $437 \mathrm{p}$.

ORGANIZAÇÃO PARA COOPERAÇÃO ECONÔMICA E DESENVOLVIMENTO. Manual de Oslo. 1997. Disponível em: <http:/www.finep.gov.br/images/apoio-e-financiamento/ manualoslo.pdf. $>$ Acesso em: 12 mar. 2016.

PATEL, P.; VEGA, M. Patterns of internationalization of corporate technology: location vs home country advantages. Research Policy, v. 28, n. 2-3, p. 145-55, 1999.

PAVITT, K. Sectoral patterns of technical change: towards a taxonomy and a theory. Research Policy, v. 13, n. 6, p. 343-373, 1984.

IBGE. Pesquisa Nacional de Inovação Tecnológica. Brasília, DF: IBGE, 2005. Disponível em: <http:/www.pintec.ibge.gov.br/>. Acesso em: 25 jul. 2016.

ROSSATO, M. V. Qualidade ambiental e qualidade de vida nos municípios do estado do Rio Grande do Sul. 2006. 168 f. Tese (Doutorado em Economia Aplicada)- Universidade Federal de Viçosa, Viçosa, MG, 2006.

SOARES, A. C. L. G. et al. Índice de desenvolvimento municipal: hierarquização dos municípios do Ceará no ano de 1997. Paraná Desenvolvimento, n. 97, p. 71-89, 1999.

SCHUMPETER, J. A. Capitalismo, socialismo e democracia. Rio de Janeiro: Fundo de Cultura, 1961. $512 \mathrm{p}$.

. Teoria do desenvolvimento econômico. São Paulo: Abril Cultural, 1982.

Recebido em: 13/11/2012.

Aceito em: 12/01/2017. 